\section{La urgencia bajo la lupa: una revisión de la producción científica sobre servicios de emergencia en hospitales desde la etnografía}

\author{
Emergency care under the magnifying glass: a \\ review of ethnographic studies in the scientific \\ literature on hospital emergency services
}

Anahi Sy 1,2

Brenda Moglia 1,2

Gisele Aragunde

Paula Derossi 1

doi: 10.1590/0102-311X00026120

\title{
Resumen
}

En este trabajo se presenta una revisión de la literatura científica de estudios etnográficos sobre los servicios de emergencias en hospitales, con el objetivo de sistematizar las investigaciones y los principales hallazgos de los trabajos, que se refieren al proceso de salud-enfermedad-atención-cuidado en servicios de emergencia o guardia hospitalaria, desde una perspectiva etnográfica. En este sentido, se realizó una revisión bibliográfica integradora de textos publicados en revistas indexadas nacionales e internacionales y en las siguientes bases electrónicas: PubMed, BVS, Scopus, Redalyc y SciELO. El corpus de análisis quedó conformado por un total de 69 artículos, a los cuales se aplicó análisis de contenido, habiendo identificado las siguientes dimensiones de análisis: calidad de atención, comunicación y vínculos, subjetividad, aplicación de tecnologías informáticas, reflexión metodológica, experiencias y prácticas de los usuarios, toma de decisiones y violencias. Los resultados permitieron identificar un proceso que se aleja de lo normativo y protocolizado, donde aspectos subjetivos del trabajador, la comunicación y las relacionales interpersonales, asi como las condiciones de trabajo, modelan, orientan y condicionan la atención y cuidado que se provee en el espacio del hospital. De este modo, se destaca el abordaje de los aspectos subjetivos en las investigaciones en salud, no sólo para comprender las perspectivas y experiencias de los trabajadores, sino para entender los obstáculos que persisten en el momento de proveer una mejor calidad de atención, complejizando un problema ignorado en la mayor parte de los análisis.

Servicios Médicos de Urgencia; Etnografía; Investigación Cualitativa

\author{
Correspondencia \\ A. Sy \\ Instituto de Salud Colectiva, Universidad Nacional de Lanús. \\ General Villegas 774, Buenos Aires 1826, Argentina. \\ anahisy@gmail.com \\ 1 Instituto de Salud Colectiva, Universidad Nacional de Lanús, \\ Buenos Aires, Argentina. \\ 2 Consejo Nacional de Investigaciones Científicas y Técnicas, \\ Buenos Aires, Argentina.
}




\section{Introdución}

Una primera aproximación a la bibliografía disponible sobre hospitales, desde la investigación cualitativa, permite visualizar que la mayor parte de los estudios, en el ámbito de la salud, parten de la perspectiva de los usuarios de los servicios, sus percepciones, las expectativas y el grado de satisfacción por la atención recibida 1,2, así como la relación y comunicación médico-paciente 3,4,5 y, asociado a ello, con frecuencia, se indaga sobre la violencia institucional 6,7,8,9,10,11. Otra línea de trabajo bastante desarrollada ha sido la formación médica en la residencia de los hospitales públicos, en particular desde la etnografía podemos citar en Estados Unidos a Byron Good 12 y en Argentina los trabajos de Bonet 13 y Ferrer 14, si bien los tres investigan desde una perspectiva etnográfica en el hospital, el foco se coloca en el proceso de formación médica en la residencia.

Etnografías de instituciones hospitalarias veremos que se remontan a la escuela de Chicago, el trabajo de Goffman, "Internados" 15, resulta revelador en ese sentido, en su mayoría realizadas en instituciones psiquiátricas 16,17,18,19. En el ámbito de hospitales generales, podemos citar numerosos trabajos a nivel mundial 20,21,22,23,24,25,26,27,28,29,30,3,32,33 y para Latinoamérica 34,35,36,37,38,39,40,41,42,43 observamos que, si bien hay trabajos en Argentina y México, la mayoría se han desarrollado en Brasil. De la totalidad analizada, sólo algunos trabajos se refieren de manera aislada a la perspectiva de los trabajadores. Al respecto, coincidimos con Graça Carapinheiro 44 quien plantea que la mayoría de los estudios en el tema parten de dos principios fundamentales, el primero que remite al hospital como estructura de cuidados, para servir a los enfermos y, el segundo, que supone las buenas relaciones humanas como clave en la mejora y eficacia de los mecanismos de la institución. Subyace a estos postulados cierta instrumentalidad organizativa y social.

El interés particular de este artículo surge de los resultados preliminares de nuestra investigación etnográfica, desarrollada en el servicio de emergencias de un hospital público de la provincia de Buenos Aires, que coloca el foco en la perspectiva de los trabajadores. Esto nos permite identificar dicho ámbito como un espacio en el que convergen las problemáticas y demandas emergentes y/o coyunturales de la población de menores ingresos, con la exigencia de responder a las llamadas urgencias y emergencias médicas, desde lo establecido (normado y/o regulado) para este servicio del hospital. En relación con esto, se puede señalar que la demanda no siempre coincide con aquello que la institución norma y regula como emergencia y/o urgencia médica; tampoco con la expectativa y proceso de trabajo cotidiano del personal médico-asistencial. Por ello, este trabajo se orienta a indagar sobre las tensiones y contradicciones que se encuentran condensadas en el espacio del servicio de emergencias médicas, y serán determinantes de la percepción de quienes trabajan en hospitales sobre su proceso de trabajo.

En ese sentido, se considera relevante el trabajo de realizar una búsqueda sistemática que presentamos bajo la forma de una revisión integradora 45 de la literatura científica publicada en revistas científicas indexadas en bases de datos internacionales, a fin de elaborar una descripción de lo producido sobre el tema.

\section{Metodología}

Para la elaboración de este artículo se realiza una revisión de la bibliografía, orientada a identificar textos de autores nacionales e internacionales que mencionan el proceso de salud-enfermedadatención-cuidado (PSEAC), en servicios de emergencia o guardia hospitalaria, desde una perspectiva etnográfica.

Cuando hablamos de PSEAC nos referimos a un proceso social que opera en todas las sociedades e involucra una gran cantidad de simbolizaciones y representaciones colectivas que tratan de dar cuenta de los saberes y formas de prevenir, tratar, controlar, aliviar y/o curar determinados padecimientos 45,46,47. Al concepto de PSEA le incorporamos la categoría de cuidado, definida como un proceso continuo y abierto, sin límites definidos que involucra a un equipo (de profesionales, máquinas, medicamentos, cuerpos, usuarios y otras personas relevantes), el cual dispone de una serie de tareas diarias para lograr una mejora en el padecimiento 48. En el campo de la salud colectiva, Ayres 49 desarrolla la 
noción de cuidado vinculada a la humanización de la atención, donde la intervención apunta ir más allá de lo técnico-normativo.

Entre los diversos tipos de revisiones, se decidió trabajar de acuerdo con la propuesta metodológica de la revisión integradora de la literatura. Esta aproximación permite la obtención, la identificación, el análisis y la síntesis de las publicaciones que son referentes de un tema determinado 50,51. Se caracteriza por la amplitud, en relación a las fuentes de datos, lo cual favorece una comprensión integral del problema a abordar, contribuyendo al desarrollo de nuevas conjeturas, hipótesis y teorias 51,52.

En el caso particular de esta revisión, procedimos de acuerdo con las cinco etapas que proponen Whittemore \& Knafl ${ }^{51}$ para llevar adelante una revisión integradora. La primera etapa consistió en delimitar el problema de investigación, siendo fundamental para orientar de forma clara la revisión (tal como lo hemos presentado en la introducción del artículo). La segunda etapa se refiere a las estrategias de búsquedas, delimitando las bases y los términos a utilizar. La tercera se vincula con la evaluación de los datos obtenidos, esto es, la rigurosidad teórico-metodológica -en nuestro caso que sean trabajos efectivamente basados en métodos etnográficos- y la relevancia de los datos que obtienen (los resultados de la segunda y tercera etapa se presentan en este apartado metodológico). La cuarta etapa es la de análisis, donde se comparan los datos extraídos para poder clasificarlos y agruparlos, se realiza una codificación en categorías, de acuerdo con el análisis de contenido, recurriendo al método de la comparación constante para relacionar, elaborar hipótesis y adquirir un nivel de abstracción mayor de las categorías codificadas. La quinta y última etapa consiste en la elaboración de una presentación de los resultados obtenidos en la revisión (la información que resulta de la cuarta y quinta etapa fue desarrollada en los apartados de resultados), donde se presentan las categorías de análisis que resultan de la codificación y una síntesis de los principales argumentos hasta algunas consideraciones finales sobre el tema.

Como parte de la segunda etapa, se realizaron búsquedas, entre los meses de mayo y junio de 2019, en las siguientes bibliotecas electrónicas: Biblioteca Virtual en Salud (BVS), PubMed, Red de Revistas Científicas de América Latina y el Caribe, España y Portugal (Redalyc), Scientific Electronic Library Online (SciELO) y Scopus. En el Cuadro 1 se presenta una descripción del alcance de cada una de ellas.

Inicialmente, se realizaron algunas búsquedas exploratorias, con diversas palabras clave/descriptores $/ \mathrm{MeSH}$, realizando una evaluación preliminar de los resultados, a fin de delimitar las palabras clave/MeSH y/o descriptores más adecuados para obtener los resultados más afines a nuestro interés. En ese sentido, quedan conformadas las búsquedas con términos libres, a excepción de PubMed, donde se incluyen MeSH y términos libres (por cuestiones técnicas y de disponibilidad del recurso). En todas las bibliotecas se hacen las búsquedas de los términos en inglés, ya que la etapa exploratoria había mostrado que se obtenían más resultados de esta manera. El campo de búsqueda, incluye título, resumen y palabras clave, con variantes y/o limitaciones propias de cada una de las bases, tal como aparece descrito en el Cuadro 2. Cabe aclarar que no se delimitó algún periodo de tiempo particular para las búsquedas de los artículos, ya que nuestro interés temático (que se trate de metodologías etnográficas desarrolladas en el ámbito de la emergencia en hospitales) fue el criterio que primó en la construcción del corpus.

$\mathrm{Al}$ aplicar este procedimiento se recupera un total de 156 artículos, de los cuales 48 son eliminados por estar duplicados. Se establece como criterio de inclusión que se trate de artículos científicos que aborden el espacio del hospital, desde una perspectiva etnográfica, antropológica y/o desde las ciencias sociales (que utilice metodología y técnicas propias de la etnografía).

Luego de la lectura del título y resumen de cada uno, se establecen algunos criterios de exclusión: (1) artículos que no estén situados en el ámbito de la guardia o emergencias médicas de hospitales. (2) artículos que, aún situándose en el espacio de la guardia/emergencia de hospital, abordan eventos de salud adversos y/o algún problema de salud particular (ej. suicidio, alcoholismo y/o accidentes entre otros). Cabe aclarar que no se delimitó ningún criterio respecto al personal de salud -profesionales o no- que pudieran incluir los análisis.

Con estos criterios, inicialmente fueron excluidos 25 artículos y luego de la lectura a texto completo se eliminaron 13 más. Es así que el corpus total de artículos analizados es de 69 (Figura 1). Todos realizan aproximaciones metodológicas cualitativas de tipo etnográficas, incluyendo como técnicas: observaciones participantes, entrevistas, estudios de caso y conversaciones informales. 


\section{Cuadro 1}

Área de cobertura y especificidad temática de las bibliotecas electrónicas seleccionadas.

\begin{tabular}{|l|c|c|}
\hline Biblioteca electrónica & Área de cobertura & Especificidad temática \\
\hline BVS & América Latina y Caribe & Ciencias de la Salud \\
\hline PubMed & América Latina y Caribe, España y Portugal & Ciencias de la Salud \\
\hline Redalyc & América Latina y Caribe, España, Portugal y Sudáfrica & Multidisciplinaria \\
\hline SciELO & Global & Multidisciplinaria \\
\hline Scopus & Multidisciplinaria \\
\hline
\end{tabular}

Fuente: elaboración propia.

\section{Cuadro 2}

Tipo de términos, campo de búsqueda y número de artículos hallados en las bibliotecas electrónicas seleccionadas.

\begin{tabular}{|c|c|c|c|c|}
\hline $\begin{array}{l}\text { Biblioteca } \\
\text { electrónica }\end{array}$ & Tipo de términos & Términos usados & $\begin{array}{l}\text { Campo de } \\
\text { búsqueda }\end{array}$ & $\begin{array}{l}\text { Artículos hallados } \\
\text { (n) }\end{array}$ \\
\hline BVS & Términos libres & Health Care; Ethnography; Emergency; Hospital & $\begin{array}{l}\text { Título, resumen y } \\
\text { asunto }\end{array}$ & 50 \\
\hline \multirow[t]{2}{*}{ PubMed } & \multirow[t]{2}{*}{$\begin{array}{l}\text { MeSH terms/Términos } \\
\text { libres }\end{array}$} & $\begin{array}{l}\text { Health Care Category; Emergency Health; Services; } \\
\qquad \text { Administration Hospital }\end{array}$ & MeSH & \multirow[t]{2}{*}{24} \\
\hline & & Ethnography & Título y resumen & \\
\hline Redalyc & Términos libres & Ethnography; Hospital & Palabras-clave & 5 \\
\hline SCIELO & Términos libres & Hospital Emergency; Ethnography & Resumen & 6 \\
\hline Scopus & Términos libres & "Health Care"; Emergency; Hospital; Ethnography & $\begin{array}{l}\text { Título, resumen y } \\
\text { palabras-clave }\end{array}$ & 71 \\
\hline
\end{tabular}

Fuente: elaboración propia a partir del procedimiento seguido en cada búsqueda.

Con respecto al momento de análisis, se llevó a cabo una primera lectura del título y resumen, a fin de realizar una clasificación preliminar de los artículos en diferentes categorías que, con la lectura del texto completo fue modificada y reelaborada hasta llegar a las categorías definitivas: "calidad de atención"; "comunicación y vínculos", ya sea entre el personal de salud o de éstos con los trabajadores; "subjetividad de los trabajadores"; "tecnologías informáticas"; "metodologías cualitativas", "toma de decisiones", "experiencias y prácticas de los usuarios" y "violencia" (de acuerdo a lo planteado para la tercera etapa de la revisión integradora).

Cuando hablamos de "subjetividad" remitimos Sherry Ortner 53, quien propone que existe una configuración cultural de las subjetividades en un mundo social atravesado por relaciones de poder desiguales; e involucra modos de percepción, afecto, pensamiento, deseo o temor, que animan a los sujetos actuantes y que se vinculan a las formaciones culturales y sociales que modelan, organizan y generan determinadas "estructuras de sentimiento" 53 (p. 25). Cuando hablamos de cultura referimos a la construcción de sentido que se da a través de procesos simbólicos que habilitan una visión del mundo compartida por los miembros de un determinado grupo social 53. 
Figura 1

Diagrama del proceso de selección de las referencias bibliográficas que conforman el corpus de investigación.

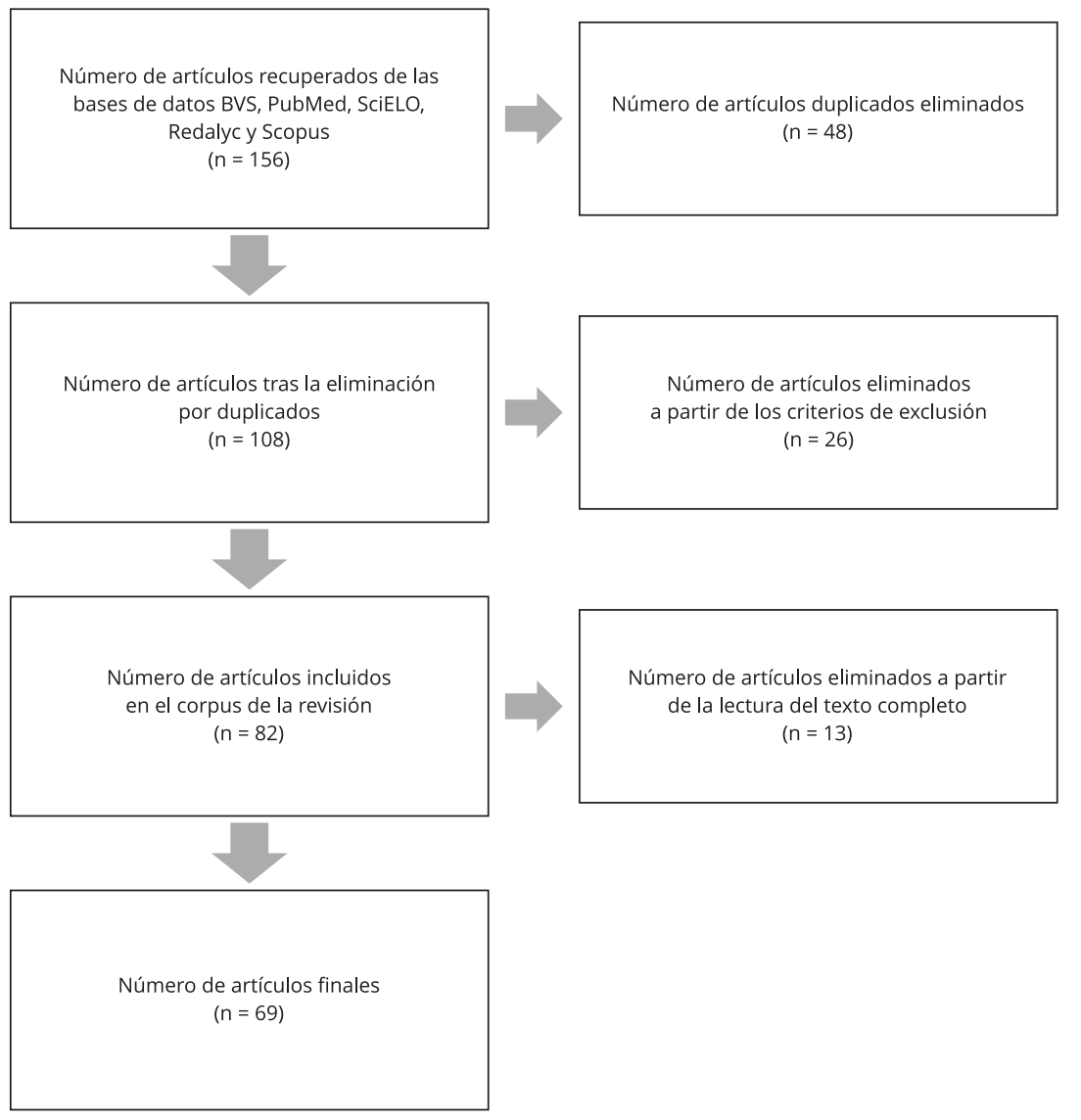

Fuente: elaboración propia.

Respecto al proceso de categorización y análisis, cabe aclarar que, además de la definición de cada una de las categorías, se realizaron controles cruzados de la clasificación y análisis en cada una de las instancias establecidas, habiendo trabajado y consensuado los mecanismos de análisis entre las cuatro autoras del presente artículo.

Por último, debe señalarse que la revisión integradora tiene limitaciones, vinculadas a las estrategias de búsquedas, que limita los hallazgos a revistas científicas indexadas (dejando fuera libros u otros formatos, donde se publica gran parte de producción etnográfica). En ese sentido, advertimos que no se trata de la totalidad de la producción sobre el tema abordado, sino de un recorte que permite construir un corpus de cierta homogeneidad para el análisis. En ese sentido, se constituye en un buen punto de partida para elaborar una síntesis de una parte del conocimiento científico producido sobre un tema de investigación, trazando un panorama que permita visualizar oportunidades para investigaciones futuras 45 . A continuación, se presentan los resultados de la clasificación y análisis de los artículos (de acuerdo con la cuarta etapa de análisis descrita). 


\section{Resultados}

Una primera lectura y análisis de los trabajos permite identificar algunas características generales para todos, que en alguna medida son coherentes con los criterios de búsqueda establecidos.

En primer lugar, todas las investigaciones toman el servicio de guardia o emergencia médica como lugar y, de una u otra forma, basan su investigación en la Etnografía como estrategia o recurso metodológico, si bien no hallamos "etnografías" en un sentido estricto. Cuando se refiere a la metodología etnográfica, esta incluye el trabajo de campo, la realización de observaciones y entrevistas, en algunos casos, además, se incluye otras técnicas adicionales como grupos focales, espacios de discusión y comunicación de resultados, entre otras variantes posibles.

La mayoría de los trabajos analizados presentan además una reflexión sobre el valor y los aportes de la aproximación y metodología etnográfica para producir datos que aporten a la atención en salud, así como destacan la posibilidad que brinda esta aproximación para visibilizar datos que no se obtendrán mediante análisis cuantitativos.

La aparente homogeneidad metodológica y técnica, desplegada en un ámbito singular, como el de la emergencia o guardia, contrasta con la diversidad temática y de países que hallamos en los estudios analizados que conforman el corpus.

La mayor parte de las investigaciones pertenece a países de habla inglesa, Estados Unidos, Australia y al Reino Unido; en mucha menor proporción Brasil y Canadá (Tabla 1). El resto de los países no presentan más de un trabajo. Respecto a las producciones australianas, cuando indagamos al respecto encontramos que se trata de uno de los países con mayor porcentaje de PBI per cápita invertido en Ciencia y Tecnología, equivalente al de Estados Unidos y superior al de Reino Unido 54. En cuanto a la temática, la mayoría aborda la comunicación y los vínculos entre el personal de salud o la calidad de atención. Australia es el país que condensa la mayor proporción de artículos sobre estas categorías. Le siguen aquellos estudios que tratan sobre aspectos subjetivos del trabajo en salud (Tabla 2).

Es interesante destacar que Estados Unidos es el único país que presenta producciones de todas las categorías descritas, y es el que más investigaciones tiene en relación a tecnologías informáticas aplicadas a la salud. Mientras que en Reino Unido se destacan las investigaciones en torno a la calidad

Tabla 1

Cantidad de artículos y porcentaje según país de publicación.

\begin{tabular}{lcc}
\hline País & \multicolumn{2}{c}{ Artículos } \\
& $\mathbf{n}$ & $\%$ \\
\hline Australia & 16 & 23,19 \\
Estados Unidos & 14 & 20,29 \\
Reino Unido & 13 & 18,84 \\
Brasil & 6 & 8,70 \\
Canadá & 5 & 7,25 \\
Varios países & 5 & 7,25 \\
Países Bajos & 2 & 2,90 \\
Sudáfrica & 2 & 2,90 \\
Noruega & 1 & 1,45 \\
Italia & 1 & 1,45 \\
Suecia & 1 & 1,45 \\
Finlandia & 1 & 1,45 \\
Argentina & 1 & 1,45 \\
No accesible & 1 & 1,45 \\
Total general & 69 & 100,00 \\
\hline
\end{tabular}

Fuente: elaboración propia. 
Tabla 2

Cantidad de artículos y porcentaje según categorías de análisis.

\begin{tabular}{lcc}
\hline Tema central & \multicolumn{2}{c}{ Artículos } \\
& $\mathbf{n}$ & $\%$ \\
\hline Calidad de atención & 13 & 18,90 \\
Comunicación y vínculos & 13 & 18,90 \\
Subjetividad & 12 & 17,40 \\
Tecnologías informáticas & 7 & 10,14 \\
Metodología cualitativa en salud & 7 & 10,14 \\
Experiencias y prácticas de los usuarios & 6 & 8,70 \\
Toma de decisiones & 6 & 8,70 \\
Violencia & 5 & 7,25 \\
Total & 69 & 100,00 \\
\hline
\end{tabular}

Fuente: elaboración propia a partir de los resultados.

de atención y a la toma de decisiones, siendo el país que concentra la mayor proporción de artículos sobre estos temas.

En el resto de los países, las producciones son mucho más escasas y los temas diversos, no pudiendo identificar regularidades.

A continuación, presentamos una descripción de los artículos analizados para cada una de las dimensiones de análisis identificadas.

\section{Calidad de atención}

Incluye aquellos artículos cuyo tema central es el estudio de los procesos de trabajo que contribuyen y/u obstaculizan la atención en el espacio de las emergencias.

Sobre aquellos factores que pueden afectarlos, un tema recurrente es el de "las interrupciones", que se refiere a la discontinuidad en el proceso de trabajo, a consecuencia de la interferencia de los usuarios, el mismo personal de salud y/o por falta de insumos o recursos 55 .

Otro aspecto que afecta a la atención es el llamado "efecto fin de semana", que indica que existe un mayor riesgo de muerte al atenderse por guardia los fines de semana, antes que durante la semana 56 .

Respecto al abordaje de cuestiones que pueden mejorar la calidad de atención, se remite a las variaciones o la mejora en los circuitos de atención en la guardia, orientados a aumentar la eficiência 57,58 o en la supervisión de tareas de enfermería que tendrían un impacto en la seguridad del paciente, así como del personal 59. A propósito de servicios de emergencia puntuales, como el de guardia obstétrica 60 , se plantea además el uso de pulsera como herramienta de identificación de los pacientes 61 o programas de atención centrados en la persona 62 . También se aborda el sistema de clasificación en enfermería como herramienta en la toma de decisiones en los servicios de emergência 63.

Otros autores colocan el foco sobre el modo en que opera el tiempo de espera en la calidad de atención brindada 64,65,66 o las dimensiones de espacio, tiempo y poder en la atención hospitalaria 67 . En otros casos, sobre la ética del cuidado cotidiano en la emergencia del hospital, analizada en términos de una "ética de cuidado" 68.

\section{Comunicación y vínculos}

Esta categoría reúne artículos centrados en las relaciones que se establecen entre los trabajadores de los servicios de guardia, donde se destaca lo relacional como una dimensión central en los procesos de trabajo en hospitales, que afecta significativamente los procesos de atención y cuidado. Se destaca que el servicio de guardia tiene sus propias creencias, lógica y valores subyacentes, que van más allá de lo que se escribe como valores organizacionales. 
Los artículos agrupados en esta categoría pueden distribuirse en tres grupos, el primero reúne aquellas investigaciones centradas en las relaciones entre los equipos de salud, analizando de qué modo pueden favorecer o no las prácticas interprofesionales orientadas a una mayor seguridad del paciente 69,70. Asimismo, se abordan los alcances y las limitaciones que generan el trabajo en equipos interprofesionales 71,72 y la influencia del equipo en el traslado y continuidad de tratamiento en otros servicios 73,74 .

Un segundo grupo de artículos explora los roles desempeñados por determinados trabajadores y cómo éstos configuran ciertas relaciones, responsabilidades y prácticas de atención, indagando sobre el trabajo de las enfermeras 75 y los médicos del departamento de emergências 76,77 .

Un tercer grupo, aborda los procesos de comunicación entre profesionales de diferentes disciplinas, centrados en el manejo de medicamentos 78,79 o acerca de las derivaciones de pacientes desde el departamento de emergencia hacia otros servicios ${ }^{80}$. En estos trabajos se observa cómo la forma de comunicar o los marcos interpretativos que utilizan los trabajadores tienen consecuencias en la seguridad del paciente, en la calidad y en la eficiencia de su atención.

\section{Subjetividad}

Esta categoría reúne aquellos artículos centrados en las experiencias, percepciones y emociones de los trabajadores, en relación a los procesos de atención y cuidado. En todos los casos se destaca la importancia de la subjetividad de los trabajadores de la salud a la hora de analizar sus prácticas y mejorar la calidad de atención. En este sentido, podemos reunir los artículos en dos grupos; por un lado, aquellos que remiten a los efectos de las condiciones de trabajo sobre los trabajadores: sentimientos de angustia, miedo, estrés o gratificación, entre otros. Por el otro, artículos que describen la influencia de los sentimientos, creencias, valores, costumbres, sobre los procesos de atención, ya sea como potenciadores u obstaculizadores de dichos procesos.

Respecto a los primeros, algunos exploran los sentimientos asociados a trabajar cotidianamente con situaciones de muerte 81,82 o con pacientes con secuelas crónicas 83 . Otro grupo de artículos remite a los sentimientos asociados a las condiciones de trabajo, se explora cómo las características de la guardia hospitalaria, tales como la sobredemanda, la urgencia, el cambio constante de prioridades, la presión, el desajuste entre la atención que desean brindar y lo que realmente logran; generan angustia y sufrimiento en los trabajadores $84,85,86$ y muchos de ellos reflexionan sobre la necesidad de analizar las diferentes emociones derivadas del trabajo para promover mejoras en la gestión y la calidad de atención 27,85 .

El segundo grupo de artículos se orienta a mostrar de qué forma esos sentimientos, creencias, valores y cultura inciden en la atención que brindan a los usuarios. Aquellos que toman el concepto de cultura analizan el espacio de la guardia como una cultura diferente a la de otros departamentos del hospital, lo cual incide de manera particular en los procesos de trabajo, en la toma de decisiones y en la calidad de atención 87,88,89. Asimismo, hallamos artículos que analizan cómo los estigmas de los que son portadores los usuarios o ciertos grupos sociales 90 y los marcos interpretativos 91 de los médicos influyen en su manera de actuar profesionalmente.

\section{Tecnologías informáticas}

Esta categoría reúne artículos en los que se presenta resultados de investigaciones sobre la aceptación, aplicación o revisión de recursos tecnológicos virtuales en instituciones de salud. Se destaca en dichos trabajos la integración de diversas disciplinas como la informática, la administración, la estadística y las ciencias sociales, cognitivas y biomédicas.

El objetivo que plantean estas investigaciones es mejorar la calidad de atención de los usuários 92 , en algunos casos, mediante la incorporación del registro electrónico de la historia clínica como una forma de asegurar la información y garantizar la seguridad del paciente 93,94 o como forma de expandir la utilidad del registro electrónico de resultados de estudios complementários 94 , y con el potencial de reutilizar los datos secundarios de la historia clínica como un sistema de control en la atención 95 . Aparece plasmada en estos trabajos la discusión sobre el acceso a la información disponible, para quiénes y de qué manera. 
Otro grupo de trabajos colocan el foco en el uso de sistemas informáticos para la toma de decisiones, traspaso de pacientes entre unidades de atención 92,94, interfase de comunicación y derivaciones prehospitalarias 96 .

Por último, algunas investigaciones indagan sobre las tecnologías ya instaladas, para implementar mejoras y adaptar el sistema, mediante seguimiento de los operadores y equipos de salud para lograr su aceptación y su uso adecuado 96,97. A diferencia de los anteriores, el trabajo de Tang et al. 97 realiza un estudio comparativo entre un sistema informático caído en desuso por parte del personal de salud y las estrategias para lograr la aceptación y continuidad en la incorporación de uno nuevo. En la mayoría de estos trabajos se destaca el lugar que ocupa la subjetividad, iniciativa y cultura de los trabajadores en el uso y toma de decisiones sobre este tipo de tecnologías.

\section{Metodología cualitativa en salud}

Esta categoría reúne aquellos artículos que remiten a la aplicación de la metodología y/o técnicas de la etnografía en el trabajo de campo, en particular cuando se trata de hospitales y/o de la guardia médica en dicho ámbito.

Algunos de ellos focalizan la discusión y desarrollo sobre la especificidad de la metodología cualitativa y/o de las técnicas etnográficas para la comprensión de las prácticas de cuidado, toma de decisiones e intervenciones en salud 98,99,100,101. Otros analizan cuestiones éticas, en algunos casos asociadas a las dificultades para obtener permisos de ética para el desarrollo de este tipo de investigaciones 102, y en otros sobre la reflexividad de aspectos que afectan a quienes desarrollan las investigaciones que, en muchos casos pueden ejercer el doble rol de investigador/ trabajador del hospital 103,104.

\section{Experiencias y prácticas de los usuarios}

Los trabajos incluidos en esta categoría, en su mayoría, exploran los patrones de comportamiento y/o percepciones de los usuarios, familiares o acompañantes en servicios de guardia de hospitales. Algunos de ellos toman pacientes críticamente enfermos 105,106,107, otros los vínculos con los profesionales 108, así como el uso de la guardia por motivos que no revisten urgencia en diversos grupos poblacionales como niños $109 \mathrm{y} / \mathrm{o}$ poblaciones indígenas 110 . En este apartado, el número de artículos fue considerablemente reducido, dado que muchos fueron excluidos al tratar problemáticas de salud puntuales (tal como fuere expresado en los criterios de exclusión).

\section{Toma de decisiones}

Los artículos incluidos en esta categoría analizan el modo en que los trabajadores, utilizando como base las reglas o protocolos de actuación establecidos por la institución, tienen capacidad de agencia y actúan en base a su experiencia, juicios y conocimientos tácitos en las elecciones y acciones que realizan en el proceso de atención.

Los artículos reflejan cómo la práctica médica se enmarca dentro de una estructura -formada por políticas, protocolos, normas, jerarquías, saberes, modos de organización institucional- que la moldea, pero no la determina por completo. Existe una agencia por parte de los trabajadores que los lleva a tomar decisiones, basadas en su experiencia y en su conocimiento tácito, cuando su juicio no coincide con lo establecido, cuando el protocolo -generado a partir del "deber ser" de la realidad supuesta-, no coincide con la realidad concreta del servicio de emergencia. Ejemplos de ello son estudios sobre el proceso de toma de decisiones relacionadas con la derivación de pacientes hacia diferentes centros de assistência 111 con la priorización de casos a atender 112,113 y con la organización y los procedimientos a utilizar 114,115,116. Al respecto se plantea que tanto los médicos como los enfermeros poseen un amplio margen de acción en dicho proceso, eso pone de manifiesto la imposibilidad de predecir todas las prácticas; el poder que tienen los trabajadores de ir transformando las prácticas de cuidado y atención, además de instituir nuevas formas de acción y la necesidad de investigar cómo se realiza ese proceso de toma de decisiones, cuál es el conocimiento implícito y las experiencias que entran en juego. 


\section{Violencias}

Esta categoría reúne aquellos artículos que abordan situaciones de agresiones, ya sean físicas como verbales, que tienen lugar en las guardias de hospitales. Los trabajos incluidos, en su mayoría, trabajan sobre violencias entre los usuarios y/o de familiares o acompañantes hacia el personal de salud 117,118,119,120. Sólo un artículo describe la violencia por parte del personal médico hacia usuarios, expresado como "cuidados inhumanos" 121. Muchos de estos trabajos usan la categoría de "riesgo", buscando identificar las posibles causas que pueden dar origen a estas situaciones 119,121, entre ellas aparecen los tiempos de espera para ser atendidos y el impacto que tiene la empatía en el proceso de atención. Algunos de estos trabajos también refieren a las percepciones y naturalización de estas violencias por parte del personal 118,120 y el registro o mejor, subregistro de tales eventos en las instituciones 117 .

\section{A modo de síntesis}

El desafío de un trabajo de revisión bibliográfica está en la posibilidad de trascender el análisis y descripción de cada artículo o conjunto de artículos, que conforman el corpus documental analizado, e ir más allá del nivel descriptivo, para generar inferencias e hipótesis de mayor alcance.

En nuestro estudio, el corpus de artículos analizados permite establecer un mapeo de temas que se han convertido en objeto de conocimiento, desde una perspectiva etnográfica en hospitales, los cuales se infiere que parten de algún problema o problematización en el ámbito de las instituciones de salud.

A este respecto, la mayor parte de las investigaciones reflexionan sobre el potencial de las aproximaciones cualitativas, en particular aquellas que colocan el foco en la metodología etnográfica, realizan consideraciones sobre el aporte teórico-metodológico de diversas técnicas y metodologías cualitativas aplicadas al campo de la salud, en particular en espacios institucionales de atención a la salud. Por un lado, se destaca el acceso a las prácticas que proveen las aproximaciones etnográficas, difíciles de captar mediante otras técnicas no intrusivas, aun cuando sean de tipo cualitativas. Por el otro, puede verse que el aporte no se reduce a lo técnico, sino que abren también la necesaria discusión sobre aspectos éticos acerca del trabajo en instituciones de salud, y en particular, aquellos que pueden derivarse de los casos en que el propio trabajador/a lleva adelante la investigación en su espacio de trabajo, con todas las implicaciones subjetivas y éticas en la producción de conocimiento que esto conlleva.

En cuanto al mapeo de temas/problemas abordados en la literatura, puede identificarse una categoría central al análisis que aparece, de diferentes modos, en todos los artículos del corpus analizado: "calidad de atención" -se entrecomillaron las categorías de análisis generales y las dimensiones de análisis identificadas al interior de las mismas quedan en cursivas. En efecto, si bien al agrupar los artículos, según el tema central de cada investigación, sólo algunos fueron incluidos en dicha categoría, la gran mayoría trabajaba algún tema/problema particular, con el fin último de aportar la calidad de atención en los hospitales.

Respecto a los artículos incluidos en "calidad de atención”, señalan la espera, los circuitos de atención, la atención centrada a las personas y las interrupciones como dimensiones centrales, si bien la espera también es una categoría que aparece asociada a los trabajos que abordan el tema de las "violencias", que en su mayoría colocan el foco en situaciones de agresiones entre usuarios o de usuarios hacia el personal de salud. Otra cuestión vinculada a la "calidad de atención" son las experiencias y prácticas de los pacientes, donde la problemática central es el uso de la guardia médica para problemas que no tienen el carácter de urgencia o emergencia. Asociado a esto puede identificarse cuestiones abordadas desde la "subjetividad" de los trabajadores de la salud, dado que los estigmas de los pacientes, los marcos interpretativos de los trabajadores, sus creencias, valores y cultura, condicionan la atención que se provee. Nuevamente aparece la "calidad de atención" en este caso asociada a la "subjetividad" del personal de salud. Al respecto, las percepciones sobre las condiciones de trabajo, la sobredemanda, la urgencia y las presiones a las que se ven sometidos genera una distancia entre la atención deseada y la brindada, lo cual se traduce en una angustia y sufrimiento que necesariamente incide en la atención brindada. Otra cuestión vinculada a la subjetividad y calidad de atención es la "comunicación 
y relaciones interpersonales"; los roles, la organización y las responsabilidades asociadas a ello, que resultan centrales.

Por otra parte, muchos artículos reflejan la distancia entre las condiciones de trabajo reales y la realidad supuesta sobre la que se asientan las políticas, protocolos y normas de las instituciones. Esta observación conduce a plantear una hipótesis interesante; la brecha entre lo supuesto (establecido y normado o protocolizado) y lo real (condiciones de trabajo y posibilidades que "no encajan" en la norma), habilita cierta creatividad y agencia en los trabajadores. Frente al deber ser, en la guardia encuentran lo efectivamente posible, que supone la toma de decisiones basada en su experiencia, criterio clínico y conocimientos tácitos. Lo interesante de este proceso es que no se trata de algo inherentemente negativo o positivo, sino que puede contribuir a la calidad de atención de manera diferente, según las particularidades de cada trabajador. En este sentido, se torna fundamental indagar en estos procesos de toma de decisiones, las motivaciones, lo coyuntural y/o circunstancial que conduce en diversas situaciones a actuar y decidir de manera autónoma, creativa, por fuera de lo normado y/o establecido en la institución.

Es así que, tanto la forma en que se toman las decisiones como los procesos comunicacionales y los vínculos, el tiempo y los circuitos de atención, el modo en que se implementa las tecnologías, y hasta los modos en que los usuarios transitan por el sistema de salud, se encuentran atravesados por esta distancia entre el deber ser o la norma y lo que efectivamente es. En ese sentido, es que pueden convertirse en elementos que mejoran o que empobrecen la calidad de atención provista, de ahí la importancia de ser investigados, utilizando metodologías cualitativas que permitan una aproximación profunda y centrada en la realidad concreta de la guardia de hospital.

\section{Comentarios finales}

El análisis realizado permite identificar un movimiento donde la subjetividad, creatividad y agencia del trabajador va moldeando los procesos de atención y cuidado, la comunicación y relaciones interpersonales, si bien éstas últimas, al mismo tiempo modelan y atraviesan la subjetividad de los trabajadores y, en este sentido, junto a las condiciones de trabajo, orientan y condicionan su creatividad y acción.

A partir de ello, podemos señalar, además, que las distancias entre lo normado y lo cotidiano, el día y la noche, la semana y el fin de semana, lo que se desea y lo que efectivamente ocurre promueve un sufrimiento capaz de producir, reproducir, así como también transformar subjetividades, es por ello que se vuelve ineludible el abordaje de los aspectos subjetivos en las investigaciones en salud. No sólo para comprender las perspectivas y experiencias de los trabajadores, sino para entender los obstáculos que persisten en el momento de proveer una mejor calidad de atención, complejizando el problema y los análisis más frecuentes.

Como tarea futura, por una parte, está el desafío de encarar un trabajo aún no abordado en Argentina, al mismo tiempo que contemplar y hacer jugar en el estudio las posibles similitudes y diferencias entre países que, a modo de hipótesis, arriesgamos más similitudes que diferencias, distancias comunes y padecimientos compartidos. Pareciera que, ante el sufrimiento, lo urgente y la inminente presencia de la muerte; independientemente de nacionalidades, políticas de salud e instituciones, los sufrimientos y problemas hallados fueran humanos y, por qué no, universales. 


\section{Colaboradores}

A. Sy participó con la idea, búsqueda, análisis, redacción y revisión del texto. B. Moglia y G. Aragunde realizarón las búsquedas, análisis y redaccióndel texto. P. Derossi colaboró en el análisis y redacción del texto.

\section{Informaciones adicionales}

ORCID: Anahi Sy (0000-0002-1281-5333); Brenda Moglia (0000-0002-3640-5232); Gisele Aragunde (0000-0002-3340-0540); Paula Derossi (00000001-6770-8220).

\section{Agradecimientos}

A la Universidad Nacional de Lanús (UNLa) por financiar y sostener el proyecto de investigación donde se enmarca este trabajo, al Instituto de Salud Colectiva (ISCo-UNLa) por favorecer un espacio de discusión e investigación académica. Al Consejo Nacional de Investigaciones Científicas y Técnicas, por el apoyo económico al trabajo de A. Sy y B. Moglia.

\section{Referencias}

1. Gill L, White L. A critical review of patient satisfaction. Leadersh Health Serv (Bradf Engl) 2009; 22:8-19.

2. Sutton LH, García RF, Hernández RA, Roche OFR. Expectativas y experiencias de los usuarios del Sistema de Salud en México: un estudio de satisfacción con la atención médica. México DF: Universidad Nacional Autónoma de México; 2013.

3. Borrott N, Kinney S, Newall F, Williams A, Cranswick N, Wong I, et al. Medication communication between nurses and doctors for paediatric acute care: an ethnographic study. J Clin Nurs 2017; 26:1978-92.

4. Aelbrecht K, Rimondini M, Bensing J, Moretti F, Willems S, Mazzi M, et al. Quality of doctorpatient communication through the eyes of the patient: variation according to the patient's educational level. Adv Health Sci Educ 2015; 20:873-84.

5. Colmenares-Roa T, Huerta-Sil G, InfanteCastañeda C, Lino-Pérez L, Alvarez-Hernández E, Peláez-Ballestas I. Doctor-patient relationship between individuals with fibromyalgia and rheumatologists in public and private health care in Mexico. Qual Health Res 2016; 26:1674-88.

6. Castro R, Erviti J. Sociología de la práctica médica autoritaria: violencia obstetricia, anticoncepción inducia y derechos reproductivos. Cuernavaca: Centro Regional de Investigaciones Multidisciplinarias, Universidad Nacional Autónoma de México; 2016. (Violencia(s), Derechos y Salud).

7. Marton B. La violencia simbólica en la consulta médica: la naturalización de la díada madrehijo y la promoción compulsiva de la lactancia materna. Sex Salud Soc 2010; (5):119-43.

8. Rueda EA. Salud, violencia estructural y ley estatutaria: un vistazo rápido a nueve patologías estructurales. Anamnenis - Revista de Bioética 2013; 8:91-5.

9. Bodelón E. Violencia institucional y violencia de género. Anales de la Cátedra Francisco Suárez 2014; 48:131-55.

10. Lopera Betancur MA. Atención de la urgencia en casa: una reacción automática. Invest Educ Enferm 2009; 27:54-9.

11. Fleury S. Salud y democracia en Brasil: valor público y capital institucional en el Sistema Único de Salud. Salud Colect 2007; 3:147-57.

12. Good B. Medicine, rationality, and experience: an anthropological perspective. New York: Cambridge University Press; 1994.

13. Bonet O. Saber e sentir: uma etnografia da aprendizagem da biomedicina. Rio de Janeiro: Editora Fiocruz; 2004. (Coleção Antropologia e Saúde).

14. Ferrer M. La maquila de médicos: una etnografía en la guardia del Hospital Nacional de Clínicas. Córdoba: Facultad de Filosofía y Humanidades, Universidad Nacional de Córdoba; 2015. 
15. Goffman E. Internados: ensayos sobre la situación social de los enfermos mentales. Buenos Aires: Amorrortu; 2012.

16. Rosenhan DL. On being sane in insane places. Science 1973; 179:250-8.

17. Álvarez Pedrosian E, Vidart D. Los estrategas del Maciel: etnografía de un hospital público. Montevideo: Universidad de la República; 2009.

18. Creswell LM. A critical black feminist ethnography of treatment for women with co-occurring disorders in the psychiatric hospital. $\mathrm{J} \mathrm{Be}$ hav Health Serv Res 2014; 41:167-84.

19. Rhodes LA. Emptying beds: the work of an emergency psychiatric unit. Berkeley: University of California Press; 1995.

20. Abadía BC, Pinilla MYA, Ariza KR, Ruiz HCS. Neoliberalism in health: the torture of the health care workers of the Bogota s Instituto Materno Infantil (child and maternity hospital). Rev Salud Pública 2012; 14:18-31.

21. Day S, Coombes RC, McGrath-Lone L, Schoenborn C, Ward H. Stratified, precision or personalised medicine? Cancer services in the 'real world' of a London hospital. Sociol Health Illn 2017; 39:143-58.

22. Duke M, Street A. Tensions and constraints for nurses in hospital-in-the-home programmes. Int J Nurs Pract 2005; 11:221-7.

23. Farre A, Cummins C. Understanding and evaluating the effects of implementing an electronic paediatric prescribing system on care provision and hospital work in paediatric hospital ward settings: a qualitatively driven mixed-method study protocol. BMJ Open 2016; 6:e010444.

24. Fassin D. The elementary forms of care: an empirical approach to ethics in a South African Hospital. Soc Sci Med 2008; 67:262-70.

25. Gaede BM. Doctors as street-level bureaucrats in a rural hospital in South Africa. Rural Remote Health 2016; 16:3461.

26. Harte JD, Sheehan A, Stewart SC, Foureur M. Childbirth supporters' experiences in a built hospital birth environment: exploring inhibiting and facilitating factors in negotiating the supporter role. HERD 2016; 9:135-61.

27. Henckes N, Nurok M. "The first pulse you take is your own" - but don't forget your colleagues. Emotion teamwork in pre-hospital emergency medical services. Sociol Health Illn 2015; 37:1023-38.

28. Horsley PA. Death dwells in spaces: bodies in the hospital mortuary. Anthropol Med 2008; 15:133-46.

29. Hughes C, van Heugten K, Keeling S, Szekely F. Being-in-the-chemotherapy-suite versus being-in-the-oncology-ward: an analytical view of two hospital sites occupied by people experiencing cancer. Cancers (Basel) 2017; 9:64.

30. Inhorn MC. Privacy, privatization, and the politics of patronage: ethnographic challenges to penetrating the secret world of Middle Eastern, hospital-based in vitro fertilization. Soc Sci Med 2004; 59:2095-108.
31. Larsen LS, Larsen BH, Birkelund R. A companionship between strangers - the hospital environment as a challenge in patient-patient interaction in oncology wards. J Adv Nurs 2014; 70:395-404.

32. Romaní O. Etnografía, técnicas cualitativas e investigación en salud: un debate abierto. Tarragona: Publicacions URV; 2013.

33. van der Geest S, Finkler K. Hospital ethnography: introduction. Soc Sci Med 2004; 59:19952001.

34. Castro EAB, Camargo Jr. KR. Por uma etnografia dos cuidados de saúde após a alta hospitalar. Ciênc Saúde Colet 2008; 13 Suppl 2:2075-88.

35. Cecchetto F. Dilemas de uma etnografia da classificação racial em espaços institucionais. Hist Ciênc Saúde-Manguinhos 2011; 18:2538.

36. Cefaï D. Provações corporais: uma etnografia fenomenológica entre moradores de rua de $\mathrm{Pa}-$ ris. Lua Nova 2010; (79):71-110.

37. Dalmolin BM, Vasconcellos M P. Etnografia de sujeitos em sofrimento psíquico. Rev Saúde Pública 2008; 42:49-54.

38. Grupo Transfuncional en Ética Clínica. Valores en medicina: etnografía de sus representaciones en un hospital de cardiología en México. Cuicuilco 2011; 18:115-32.

39. Elsen I, Monticelli M. Nas trilhas da etnografia: reflexões em relação ao saber em enfermagem. Rev Bras Enferm 2003; 56:193-7.

40. Feltrin RB, Velho L. Representações do corpo feminino na menopausa: estudo etnográfico em um hospital-escola brasileiro. Sex Salud Soc 2016; (22):148-74.

41. García MG, Recoder ML, Margulies S. Space, time and power in hospital health care: contributions based on the ethnography of an obstetric center. Salud Colect 2017; 13:391-409.

42. Morais AC, Camargo CL, Quirino MD. A etnografia nas pesquisas de enfermagem com ênfase no cuidado. Cogitare Enferm 2011; 16:549-55.

43. Oliveira M J. Uma etnografia sobre o atendimento psicoterapêutico a transexuais. Revista Estudos Feministas 2014; 22:839-62.

44. Carapinheiro G. Saberes e poderes no hospital: uma sociologia dos serviços hospitalares. Porto: Edições Afrontamento; 1993. (Colecção Saber Imaginar o Social).

45. Menéndez EL. La enfermedad y la curación ¿Qué es medicina tradicional? Alteridades 1994; 4:71-83.

46. Menéndez EL. Modelos de atención de los padecimientos: de exclusiones teóricas y articulaciones prácticas. Ciênc Saúde Colet 2003; 8:185-207.

47. Menéndez EL. Las enfermedades ¿ son solo padecimientos?: biomedicina, formas de atención "paralelas" y proyectos de poder. Salud Colect 2015; 11:301-30.

48. Mol A. The logic of care: health and problem of patient choice. London: Routledge; 2008. 
49. Ayres JRCM. El cuidado: los modos de ser (del) humano y las prácticas de salud. In: Paiva V, Ayres JRCM, Capriati A, Amuchástegui A, Pecheny M, organizadores. Prevención, promoción y cuidado: enfoques de vulnerabilidad y derechos humanos. Temperley: TesseoPress; 2018. p. 1110-40.

50. Botelho LLR, Cunha CC A, Macedo M. O método da revisão integrativa nos estudos organizacionais. Gestão \& Sociedade 2011; 5:121-36.

51. Whittemore R, Knafl K. The integrative review: updated methodology. J Adv Nurs 2005; 52:546-53.

52. Deslandes SF, Flach RMD. Abuso digital nos relacionamentos afetivo-sexuais: uma análise bibliográfica. Cad Saúde Pública 2017; 33:e0013851.

53. Ortner S. Geertz, subjetividad y conciencia posmoderna. Etnografías Contemporáneas 2005; 1:25-54.

54. Kreiner A. ¿Por qué invertir en ciencia y tecnología? Agencia TSS 2020; 31 ene. http://www. unsam.edu.ar/tss/por-que-invertir-en-cien cia-y-tecnologia/.

55. Brixey JJ, Tang Z, Robinson DJ, Johnson CW, Johnson TR, Turley JP, et al. Interruptions in a level one trauma center: a case study. Int J Med Inform 2008; 77:235-41.

56. Tarrant C, Sutton E, Angell E, Aldridge CP, Boyal A, Bion J. The "weekend effect" in acute medicine: a protocol for a team-based ethnography of weekend care for medical patients in acute hospital settings. BMJ Open 2017; $7: \mathrm{e} 016755$.

57. O'Cathain A, Knowles E, Turner J, Hirst E, Goodacre S, Nicholl J. Variation in avoidable emergency admissions: multiple case studies of emergency and urgent care systems. J Health Serv Res Policy 2016; 21:5-14.

58. Nugus P, Forero R, McCarthy S, Mcdonnell G, Travaglia J, Hilman K, et al. The emergency department "carousel": an ethnographicallyderived model of the dynamics of patient flow. Int Emerg Nurs 2014; 22:3-9.

59. Weaver SH, Lindgren TG. Getting safely through the shift: a qualitative exploration of the administrative supervisor role. J Nurs Manag 2017; 25:430-7.

60. Montigny F, Verdon C, Dubeau D, Devault A, St-André M, Tchouaket Nguemeleu E, et al. Protocol for evaluation of the continuum of primary care in the case of a miscarriage in the emergency room: a mixed-method study. BMC Pregnancy Childbirth 2017; 17:124.

61. Smith AF, Casey K, Wilson J, FischbacherSmith D. Wristbands as aids to reduce misidentification: an ethnographically guided task analysis. Int J Qual Health Care 2011; 23:5909.

62. Dellenborg L, Wikström E, Andersson Erichsen A. Factors that may promote the learning of person-centred care: an ethnographic study of an implementation programme for healthcare professionals in a medical emergency ward in Sweden. Adv Health Sci Educ 2019; 24:353-81.
63. Fry M, Stainton C. An educational framework for triage nursing based on gatekeeping, timekeeping and decision-making processes. Accid Emerg Nurs 2005; 13:214-9.

64. Melon KA, White D, Rankin J. Beat the clock! Wait times and the production of «quality» in emergency departments. Nurs Philos 2013; 14:223-37.

65. Nugus $P$, Braithwaite $J$. The dynamic interaction of quality and efficiency in the emergency department: squaring the circle? Soc Sci Med 2010; 70:511-7.

66. O’Neill E, Woodgate D, Kostakos V. Easing the wait in the emergency room: building a theory of public information systems. In: Proceedings of the 2004 Conference on Designing Interactive Systems Processes, Practices, Methods, and Techniques. http://portal.acm.org/cita tion.cfm?doid=1013115.1013120 (accedido el 05/Nov/2019).

67. García MG, Recoder ML, Margulies S. Espacio, tiempo y poder en la atención hospitalaria de la salud y la enfermedad: aportes de una etnografía de un centro obstétrico. Salud Colect 2017; 13:391-409.

68. Fassin D. The elementary forms of care. Soc Sci Med 2008; 67:262-70.

69. Collin KM, Valleala UM, Herranen S, Paloniemi S. Ways of interprofessional collaboration and learning in emergency work. Studies in Continuing Education 2012; 34:281-300.

70. Nugus P. Re-structuring the negotiated order of the hospital. Sociol Health Illn 2019; 41:378-94.

71. Webster F, Rice K, Dainty KN, Zwarenstein M, Durant S, Kuper A. Failure to cope: the hidden curriculum of emergency department wait times and the implications for clinical training. Acad Med 2015; 90:56-62.

72. Petit dit Dariel O, Cristofalo P. A meta-ethnographic review of interprofessional teamwork in hospitals: what it is and why it doesn't happen more often. J Health Serv Res Policy 2018; 23:272-9.

73. Iedema R, Ball C, Daly B, Young J, Green T, Middleton PM, et al. Design and trial of a new ambulance-to-emergency department handover protocol: 'IMIST-AMBO'. BMJ Qual Saf 2012; 21:627-33.

74. Iedema R, Merrick E. Analysing teamwork in health care: what matters when clinicians negotiate the continuity of clinical tasks and care responsibilities? Commun Med 2016; 13:8597.

75. Nugus P, Forero R. Understanding interdepartmental and organizational work in the emergency department: an ethnographic approach. Int Emerg Nurs 2011; 19:69-74.

76. Gilardi S, Guglielmetti C, Pravettoni G. Interprofessional team dynamics and information flow management in emergency departments. J Adv Nurs 2014; 70:1299-309.

77. van Schothorst J, van den Brand CL, Gaakeer MI, Wallenburg I. The role of emergency physicians in the institutionalization of emergency medicine. Eur J Emerg Med 2017; 24:301-7. 
78. Huby G, Brook JH, Thompson A, Tierney A. Capturing the concealed: Interprofessional practice and older patients' participation in decision-making about discharge after acute hospitalization. J Interprof Care 2007; 21:5567.

79. Liu W, Gerdtz M, Manias E. Creating opportunities for interdisciplinary collaboration and patient-centred care: how nurses, doctors, pharmacists and patients use communication strategies when managing medications in an acute hospital setting. J Clin Nurs 2016; 25:2943-57.

80. Nugus P, McCarthy S, Holdgate A, Braithwaite J, Schoenmakers A, Wagner C. Packaging patients and handing them over: communication context and persuasion in the emergency department. Ann Emerg Med 2017; 69:210-7.e2.

81. Aredes J S, Giacomin KC, Firmo JOA. A práxis médica no pronto atendimento diante do paciente com sequelas crônicas: culpa, temor e compaixão. Trab Educ Saúde 2018; 16:117799.

82. Aredes JDS, Giacomin KC, Firmo JOA. O médico diante da morte no pronto socorro. Rev Saúde Pública 2018; 52:42.

83. Aredes JS, Firmo JOA, Giacomin KC. A morte que salva vidas: complexidades do cuidado médico ao paciente com suspeita de morte encefálica. Cad Saúde Pública 2018; 34:e00061718.

84. Kelley ML, Parke B, Jokinen N, Stones M, Renaud D. Senior-friendly emergency department care: an environmental assessment. J Health Serv Res Policy 2011; 16:6-12.

85. Parsons K, Gaudine A, Swab M. Older nurses' experiences of providing direct care in hospital nursing units: a qualitative systematic review. JBI Database System Rev Implement Rep 2018; 16:669-700.

86. Taylor BJ, Rush KL, Robinson CA. Nurses' experiences of caring for the older adult in the emergency department: a focused ethnography. Int Emerg Nurs 2015; 23:185-9.

87. Cole E, Crichton N. The culture of a trauma team in relation to human factors. J Clin Nurs 2006; 15:1257-66.

88. Mackintosh N, Humphrey C, Sandall J. The habitus of "rescue" and its significance for implementation of rapid response systems in acute health care. Soc Sci Med 2014; 120:23342.

89. Person J, Spiva L, Hart P. The culture of an emergency department: an ethnographic study. Int Emerg Nurs 2013; 21:222-7.

90. Henderson S, Clare L, Stacey DD. Social Stigma and the dilemmas of providing care to substance users in a safety-net emergency department. J Health Care Poor Underserved 2008; 19:1336-49.

91. Hilligoss B. Selling patients and other metaphors: a discourse analysis of the interpretive frames that shape emergency department admission handoffs. Soc Sci Med 2014; 102:11928.
92. Hilligoss B, Zheng K. Chart biopsy: an emerging medical practice enabled by electronic health records and its impacts on emergency department-inpatient admission handoffs. J Am Med Inform Assoc 2013; 20:260-7.

93. Nelson P, Bell AJ, Nathanson L, Sanchez LD, Fisher J, Anderson PD. Ethnographic analysis on the use of the electronic medical record for clinical handoff. Intern Emerg Med 2017; 12:1265-72.

94. Dahm MR, Georgiou A, Westbrook JI, Greenfield D, Horvath AR, Wakefield D, et al. Delivering safe and effective test-result communication, management and follow-up: a mixedmethods study protocol. BMJ Open 2018; 8:e020235.

95. Dixon-Woods M, Redwood S, Leslie M, Minion J, Martin GP, Coleman JJ. Improving quality and safety of care using "technovigilance": an ethnographic case study of secondary use of data from an electronic prescribing and decision support system. Milbank Q 2013; 91:42454.

96. Pope C, Halford S, Turnbull J, Prichard J, Calestani M, May C. Using computer decision support systems in NHS emergency and urgent care: ethnographic study using normalisation process theory. BMC Health Serv Res 2013; 13:111.

97. Tang Z, Johnson TR, Tindall RD, Zhang J. Applying heuristic evaluation to improve the usability of a telemedicine system. Telemed J E Health 2006; 12:24-34.

98. Grigg M, Endacott R, Herrman H, Harvey C. An ethnographic study of three mental health triage programs. Int J Ment Health Nurs 2004; $13: 145-51$

99. Heasman B, Reader TW. What can acute medicine learn from qualitative methods? Curr Opin Crit Care 2015; 21:460-6.

100. Aredes JS, Firmo JOA, Leibing A, Giacomin KC. Reflexões sobre um fazer etnográfico no pronto-socorro. Cad Saúde Pública 2017; 33:e00118016.

101. Santiano N, Baramy L-S, Young L, Saggu G, Cabrera R, Parr M. Problems and solutions arising during a study in visual semantics of the medical emergency team system. Qual Health Res 2008; 18:1336-44.

102. Roberts L, Henderson J, Willis E, Muir-Cochrane E. The challenges of gaining ethics approval for ethnographic research in the prehospital setting. J Psychiatr Ment Health Nurs 2013; 20:374-8.

103. Bailey CJ. Practitioner to researcher: reflections on the journey. Nurse Res 2007; 14:1826.

104. Jacoby SF. The insight and challenge of reflexive practice in an ethnographic study of black traumatically injured patients in Philadelphia. Nurs Inq 2017; 24:e12172.

105. Brito MEM, Damasceno AK C, Pinheiro PNC, Vieira LJE S. A cultura no cuidado familiar à criança vítima de queimaduras. Rev Eletrônica Enferm 2010; 12:321-5. 
106. Hudgins A, Rising KL. Fear, vulnerability and sacrifice: Drivers of emergency department use and implications for policy. Soc Sci Med 2016; 169:50-7.

107. Barreto MS, Marcon SS, Garcia-Vivar C. Patterns of behaviour in families of critically ill patients in the emergency room: a focused ethnography. J Adv Nurs 2017; 73:633-42.

108. Olthuis G, Prins C, Smits M-J, van de Pas H, Bierens J, Baart A. Matters of concern: a qualitative study of emergency care from the perspective of patients. Ann Emerg Med 2014; 63:311-9.e2.

109. Berry A, Brousseau D, Brotanek JM, TomanyKorman S, Flores G. Why do parents bring children to the emergency department for nonurgent conditions? A qualitative study. Ambul Pediatr 2008; 8:360-7.

110. Browne AJ, Smye VL, Rodney P, Tang SY, Mussell B, O’Neil J. Access to primary care from the perspective of aboriginal patients at an urban emergency department. Qual Health Res 2011; 21:333-48.

111. Newgard CD, Nelson MJ, Kampp M, Saha S, Zive D, Schmidt T, et al. Out-of-hospital decision making and factors influencing the regional distribution of injured patients in a trauma system. J Trauma 2011; 70:1345-53.

112. Back J, Ross AJ, Duncan MD, Jaye P, Henderson K, Anderson JE. Emergency department escalation in theory and practice: a mixedmethods study using a model of organizational resilience. Ann Emerg Med 2017; 70:659-71.

113. Johannessen LEF. Beyond guidelines: discretionary practice in face-to-face triage nursing. Sociol Health Illn 2017; 39:1180-94.

114. Allard J, Bleakley A. What would you ideally do if there were no targets? An ethnographic study of the unintended consequences of topdown governance in two clinical settings. Adv Health Sci Educ Theory Pract 2016; 21:80317.
115. Brummell SP, Seymour J, Higginbottom G. Cardiopulmonary resuscitation decisions in the emergency department: an ethnography of tacit knowledge in practice. Soc Sci Med 2016; 156:47-54.

116. Franklin A, Liu Y, Li Z, Nguyen V, Johnson TR, Robinson D, et al. Opportunistic decision making and complexity in emergency care. J Biomed Inform 2011; 44:469-76.

117. Knowles E, Mason SM, Moriarty F. "I'm going to learn how to run quick": exploring violence directed towards staff in the emergency department. Emerg Med J 2013; 30:926-31.

118. Renker P, Scribner SA, Huff P. Staff perspectives of violence in the emergency department: appeals for consequences, collaboration, and consistency. Work 2015; 51:5-18.

119. Lau JBC, Magarey J, Wiechula R. Violence in the emergency department: an ethnographic study (Part I). Int Emerg Nurs 2012; 20:69-75.

120. Lau JBC, Magarey J, Wiechula R. Violence in the emergency department: an ethnographic study (Part II). Int Emerg Nurs 2012; 20:12632.

121. Shelmerdine S. Pathways to inhumane care: masculinity and violence in a South African emergency unit. SAGE Open 2017; 7:1-12. 
Abstract

The article presents a review of ethnographic studies in the scientific literature on hospital emergency services, with the objective of systematizing the studies and their principal findings, referring to the health-disease-healthcare process in hospital emergency services from an ethnographic perspective. An integrative literature review was performed of studies published in Argentine and international indexed journals and in the following electronic databases: PubMed, VHL, Scopus, Redalyc, and SciELO. The corpus of the analysis consisted of a total of 69 articles, which were submitted to content analysis, having identified the following analytical dimensions: quality of care, communication and bonds, subjectivity, application of information technologies, methodological reflection, patients' experiences and practices, decision-making, and violence. The results allowed identifying a process that differs from guidelines and protocols, in which healthcare workers' subjective aspects, communication and interpersonal relations, and working conditions shape, orient, and condition the treatment and care provided in the hospital. The article thus highlights the approach to subjective aspects in health studies, to understand not only health workers' perspectives and experiences but also the persistent barriers to providing better quality of care, complexifying a problem ignored by a large share of the analyses.

Emergency Medical Services; Ethnography; Qualitative Research

\section{Resumo}

Este trabalho apresenta uma revisão da literatura científica de estudos etnográficos sobre os serviços de emergência hospitalares, com o intuito de sistematizar as pesquisas e os seus principais achados, que tratam ao processo de saúde-doença-atençãocuidado nos serviços de emergência ou de plantão hospitalar, desde uma perspectiva etnográfica. Neste sentido, foi realizada uma revisão bibliográfica integradora de textos publicados em revistas indexadas nacionais e internacionais e nas seguintes bases de dados: PubMed, BVS, Scopus, Redalyc e SciELO. O corpus analítico foi composto por um total de 69 artigos, aos que foi aplicada uma análise de conteúdo, após a identificação das seguintes dimensões de análise: qualidade da atenção, comunicação e vínculos, subjetividade, uso de tecnologias informáticas, reflexão metodológica, experiências e práticas dos usuários, tomada de decisões e violências. Os resultados permitiram identificar um processo que se afasta das normas e dos protocolos, onde aspectos subjetivos do trabalhador, a comunicação e as relações interpessoais, bem como as condições de trabalho, moldam, orientam e condicionam a atenção e os cuidados proporcionados no ambiente hospitalar. Assim, destaca-se a abordagem dos aspectos subjetivos nas pesquisas em saúde, não apenas para entender as perspectivas e experiências dos trabalhadores, como também para entender os obstáculos que persistem na hora oferecer uma atenção de melhor qualidade, problematizando um problema ignorado na maior parte das análises.

Serviços Médicos de Emergência; Etnografia; Pesquisa Qualitativa
Recibido el 12/Feb/2020

Versión final presentada el 20/Ago/2020

Aprobado el 23/Sep/2020 